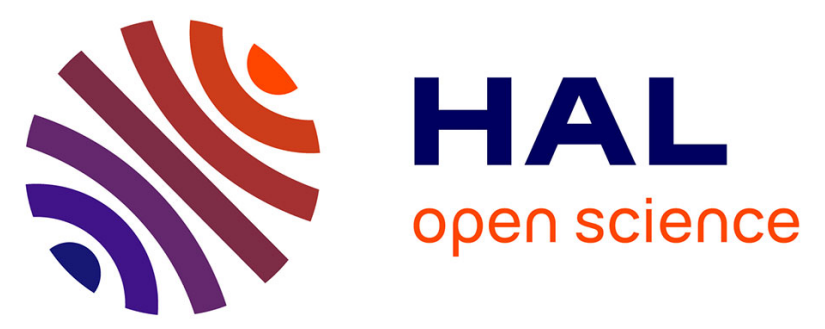

\title{
Découverte fortuite de micro-foyers de carcinome épidermoïde au sein d'ostéoradionécrose mandibulaire : quelle prise en charge?
}

M. Lesnik, O. de Crouy Chanel, M. Lefevre, S. Samaha, S. Perié, J. Lacau St-Guily, B. Baujat

\section{To cite this version:}

M. Lesnik, O. de Crouy Chanel, M. Lefevre, S. Samaha, S. Perié, et al.. Découverte fortuite de microfoyers de carcinome épidermoïde au sein d'ostéoradionécrose mandibulaire: quelle prise en charge?. Annales françaises d'Oto-rhino-laryngologie et de Pathologie Cervico-faciale, 2019, 136, pp.84 - 87. 10.1016/j.aforl.2017.09.017 . hal-03486548

\section{HAL Id: hal-03486548 \\ https://hal.science/hal-03486548}

Submitted on 20 Dec 2021

HAL is a multi-disciplinary open access archive for the deposit and dissemination of scientific research documents, whether they are published or not. The documents may come from teaching and research institutions in France or abroad, or from public or private research centers.
L'archive ouverte pluridisciplinaire HAL, est destinée au dépôt et à la diffusion de documents scientifiques de niveau recherche, publiés ou non, émanant des établissements d'enseignement et de recherche français ou étrangers, des laboratoires publics ou privés.

\section{(c) (1) $\$$}

Distributed under a Creative Commons Attribution - NonCommerciall 4.0 International 
Découverte fortuite de micro-foyers de carcinome épidermoïde au sein d'ostéoradionécrose mandibulaire : quelle prise en charge?

Maria LESNIK (1)*, Olivier DE CROUY CHANEL (1), Marine LEFEVRE (2), Sabine SAMAHA (1), Sophie PERIE (1), Jean LACAU ST GUILY (1) Bertrand BAUJAT (1)

(1) Service d'Oto-Rhino-Laryngologie et de Chirurgie Cervico-Faciale

(2) Service d'Anatomie Pathologique

Université Pierre et Marie Curie, Paris VI, Hôpital Tenon, Assistance Publique Hôpitaux de Paris , 4, rue de la Chine, 75020 PARIS, France

* auteur correspondant.

E-mail: maria.lesnik@gmail.com

Service d'Oto-Rhino-Laryngologie et de Chirurgie Cervico-faciale, Institut Curie, 26 rue d'Ulm, 75005 PARIS, France

Les auteurs déclarent n'avoir aucun conflit d'intérêt. 
Abstract

Objectifs

L'ostéoradionécrose mandibulaire (ORN) est une complication commune de la radiothérapie des voies aérodigestives supérieures (VADS). Son traitement est souvent chirurgical. Des foyers de carcinome épidermoïde (CE) peuvent être exceptionnellement découverts au sein de l'ORN lors de l'analyse anatomopathologique des pièces opératoires. Nous discutons leur prise en charge à partir d'une courte série de patients.

Matériels et méthode

Cette étude monocentrique rétrospective porte sur les patients pris en charge entre 2012 et 2014 pour ORN avec découverte fortuite de micro-foyers de CE.

Résultats

Cinq patients présentant une ORN mandibulaire au sein de laquelle a été retrouvé fortuitement un foyer microscopique de CE ont été inclus. Le délai moyen de survenue de l'ORN était de 42 mois après la fin de la radiothérapie pour CE localement avancée de la cavité buccale ou de l'oropharynx. Le traitement chirurgical était une mandibulectomie non-interruptrice ou interruptrice avec reconstruction par lambeau libre. Aucune récidive n'est survenue durant le suivi moyen de 35 [24 - 46] mois.

\section{Conclusion}

La découverte fortuite de micro-foyers de CE au sein de mandibules atteintes d'ORN est rare et non rapporté dans la littérature. Le manque de données bibliographiques ne permet pas actuellement de définir la prise en charge chirurgical optimale, mais la présente étude plaide 
pour une analyse anatomopathologique soigneuse des pièces d'ORN ainsi que d'un traitement le plus conservateur possible afin d'éviter une chirurgie trop invasive. 


\section{Introduction}

La radiothérapie a une place majeure dans l'arsenal thérapeutique des tumeurs des VADS, au sein de stratégies de prise en charge variées, associée ou non à la chirurgie ou la chimiothérapie. L'ostéoradionécrose (ORN) mandibulaire est l'une de ses complications bien connues, survenant dans un délai de 6 mois à parfois plus de 5 ans après le traitement [1-3]. Décrite pour la première fois par Regaud en 1920, elle demeure un challenge thérapeutique.

L'incidence de l'ORN varie de $0,9 \%$ à $35 \%$ selon les auteurs [4,5]. Cette variation d'incidence importante peut découler de populations étudiées non homogènes, de périodes de suivi variables et de la sélection des patients.

L'histoire habituelle d'une ORN débute typiquement par une déhiscence muqueuse avec exposition osseuse persistante au-delà de 3 mois [1,6,7]. Chez certains patients, elle se déclarera par une fracture mandibulaire pathologique sans nécessairement exposition osseuse. Son évolution se complique souvent : trismus, douleurs fréquemment neuropathiques et parfois difficiles à traiter, fistulisations, et poussées inflammatoires et infectieuses. Fractures pathologiques, séquestres osseux et orostomes rendent précaire voire impossible l'alimentation orale. De plus, ces patients subissent fréquemment l'ensemble des effets secondaires à long terme de la radiothérapie (i.e. xerostomie, trismus chronique, dysphagie, diminution de la mobilité linguale). L'ensemble de ces atteintes ont des conséquences péjoratives tant au niveau somatique que psychologique et émotionnel. Les risques de dénutrition sont importants, fragilisant encore plus la cicatrisation.

L'ORN se développe en territoire irradié, donc hypovascularisé [1,7,8] (endartérite radioinduite) avec hypocellularité de la médullaire osseuse, du périoste et de l'endothélium et diminution de production de matrice extra-cellulaire $[9,10]$. 
Les doses importantes d'irradiation, à 70Gy, et/ou les traumatismes chirurgicaux favorisent l'apparition d'ORN précoces, soit dans les deux ans suivant la fin de la radiothérapie [11-13]. Une apparition plus tardive est très souvent induite par un traumatisme dentaire au sein de tissus mandibulaires osseux hypovascularisés, paucicellulaires et hypoxiques. L'agression de cet os très fragilisé entraine une plaie chronique [3,14].

Les critères décisionnels dans la prise en charge optimale d'une ORN sont multiples [15]. Lorsqu'un traitement chirurgical est nécessaire, il peut être simple (débridement, décortication et séquestrectomie) mais également complexe, pouvant aller jusqu'à une mandibulectomie interruptrice avec reconstruction par lambeau libre [16,17]. La découverte fortuite de foyers microscopiques de CE au sein d'ORN n'est pas décrite, y compris dans les séries les plus importantes ${ }^{4}$, et la conduite à tenir dans cette situation spécifique n'est pas établie.

Nous rapportons ici une série de patients chez lesquels ont été découverts fortuitement de micro foyers de CE au sein de plus larges atteintes d'ORN mandibulaire.

\section{Matériel et méthode}

Pour cette étude monocentrique rétrospective, nous avons revu les dossiers de patients pris en charge entre 2012 et 2014 pour ORN mandibulaire et chez lesquels un foyer microscopique de $\mathrm{CE}$ a été découvert fortuitement sur pièce anatomopathologique.

Tous les patients avaient été pris en charge pour leur tumeur initiale et l'ORN secondaire dans notre centre, tant en chirurgie qu'en radiothérapie. La localisation tumorale initiale, les caractéristiques des patients, les traitements et la prise en charge de l'ORN ont été relevés. 


\section{Résultats}

Cinq patients présentant une ORN mandibulaire au sein de laquelle a été retrouvé fortuitement un foyer microscopique de CE ont été inclus (1 femme, 4 hommes) entre 2005 et 2012 pour un carcinome épidermoïde de la cavité buccale $(n=2)$ ou de l'oropharynx $(n=3)$. L'âge moyen au traitement de l'ORN était de 64 ans [53 - 73]. Le délai moyen de suivi après la tumeur initiale était de 76 mois [33 - 105]. Ces tumeurs initiales étaient localement avancées, de stade III $(n=2)$ et IV $(n=3)$. Le traitement de ces tumeurs comportait une chimiothérapie néoadjuvante $(n=4)$, suivie de chirurgie puis de radiothérapie $(n=5)$, avec chimiothérapie concomittante chez 2 patients.

Les doses de la radiothérapie allaient de 50 à 70 Gy sur le site tumoral. Le délai moyen de survenue de l'ORN après la fin de la radiothérapie était de 42 mois [12 - 60]. Table1.

Chez 3 patients, un curetage mandibulaire à visée thérapeutique a été réalisé. L'analyse histologique, systématique, a retrouvé des foyers microscopiques de CE. Après discussion en réunion de concertation pluridisciplinaire $(\mathrm{RCP})$, une reprise chirurgicale plus large a été décidée: mandibulectomie non-interruptrice $(n=2)$ ou interruptrice avec reconstruction par lambeau libre de fibula $(n=1)$. L'analyse histologique minutieuse des pièces opératoires de mandibulectomie n'a ensuite pas retrouvé de reliquat de carcinome épidermoïde, mais uniquement l'ostéoradionécrose.

Chez les 2 autres patients, le curetage a permis de confirmer une ORN. Les lésions étant très étendues, une chirurgie d'éxérèse par mandibulectomie interruptrice de l'os ostéoradionécrotique était retenue. Seul l'un des deux patients a pu bénéficier d'une reconstruction par lambeau libre de fibula. En effet, le patient 5 présentait un état général

dégradé et une $3^{\text {ème }}$ localisation tumorale (laryngée). L'analyse histologique des pièces de 
mandibulectomie a révélé des microfoyers de carcinome épidermoïde au sein de l'ORN connue.

Pour tous les patients, un suivi rapproché a été proposé par la RCP. Aucune récidive n'a été observée durant le suivi de 35 mois [24 - 46] en moyenne. Table 2.

\section{Discussion}

Les ORN sont parmi les nombreux effets secondaires de la radiothérapie des cancers des VADS, de prise en charge particulièrement difficile et complexe. Le traitement chirurgical, son importance et le moment opportun pour la pratiquer sont au coeur de la discussion de la séquence thérapeutique. Les algorithmes de traitement sont variables selon les auteurs. En cas de séquestres osseux, d'ORN étendue, ou d'inefficacité des traitements médicaux, une chirurgie est indiquée $[9,18]$. Les ORN étendues requièrent le plus souvent une mandibulectomie interruptrice avec reconstruction, si celle-ci est possible. Les taux de succès des lambeaux libres dans cette indication peuvent aller jusqu'à 98\% [19,20] malgré les tissus irradiés. Par ailleurs, la préservation, et idéalement l'amélioration, de la qualité de vie chez ces patients ayant déjà subi des traitements lourds est fondamentale, et une approche maximaliste dans le traitement de l'ORN peut malheureusement être péjorative [21]. Jacobson et al [22] ont rapporté une série de 42 patients qui, malgré un traitement chirurgical de l'ORN, parfois avec reconstruction par lambeau libre, ont gardé un trismus et un oedème persistants, le traitement chirurgical ayant néanmoins permis une sédation au moins partielle des douleurs. Malgré de nombreuses publications sur l'évaluation et la prise en charge des ORN, il existe très peu d'études randomisées, prospectives, contrôlées [9]. La plupart des études sont de petites cohortes, reflétant une pratique de centre, ne spécifiant pas toujours la durée de suivi ou l'évolution à distance de la pathologie. A notre connaissance, aucune étude ne mentionne 
de découverte fortuite à l'anatomopathologie de foyers microscopiques de carcinome épidermoïde au sein d'ORN mandibulaire.

Dans cette série, nous rapportons 5 cas de patients traités pour ORN mandibulaire et chez lesquels des foyers de carcinome épidermoïde ont été découverts fortuitement sur les pièces opératoires. La très faible incidence de ce type d'évènement empêche de grandes séries de patients, même au sein de centres comme le nôtre, spécialisés dans la prise en charge des cancers des VADS. Aucun des 5 patients n'avait présenté de tumeur au niveau du site de l'ORN: les tumeurs initiales étaient localisées au niveau de l'oropharynx chez 3 patients, et au niveau de la langue chez les 2 autres, sans extension au plancher buccal ou à la mandibule.

Tous les patients présentaient chroniquement les signes typiques mais non spécifiques de l'ORN: douleur, exposition osseuse non résolutive, trismus, infection chronique, mais aucun signe laissant suspecter une nouvelle localisation tumorale maligne. De même, l'examen clinique était rassurant sur le plan carcinologique. Cependant, une ORN et une nouvelle localisation tumorale peuvent se manifester de manière similaire et non spécifique : douleur, plaie chronique, trismus, œdème, inflammation chronique.

Dans notre série, deux patients ont nécessité une mandibulectomie interruptrice en raison de la gravité et de l'étendue de l'ORN, mais pour les 3 autres, l'ORN plus limitée était accessible à un curetage simple, et la chirurgie plus extensive et mutilante a été décidée au regard des résultats anatomopathologiques. Cependant, sur les pièces de mandibulectomie chez ces patients, aucun reliquat tumoral n'a été retrouvé, ce qui suggère qu'une attitude moins invasive aurait pu être proposée.

L'analyse histologique des pièces opératoires de cette série de patients a bien retrouvé des caractéristiques typiques d'ORN, mais des foyers microscopiques de carcinome épidermoïde ont également été constatés (Fig. 1). La présence d'atypies cellulaires permettait 
d'éliminer l'hypothèse de cellules épithéliales déplacées lors des prélèvements. Comme dans les carcinomes bien différenciés, les cellules immatures/basales étaient situées en périphérie, les cellules plus matures étant vers le centre des foci. Une néovascularisation s'était développée et les foyers de carcinome étaient présents au sein des vastes plages d'ORN, sans solution de continuité avec la muqueuse gingivale ou la peau. Le stroma adjacent était diminué et contenait un infiltrat inflammatoire (lymphocytes). Seule une analyse histopathologique soigneuse de la pièce opératoire a permis d'identifier ces micro-foyers tumoraux.

Malgré le caractère évidemment malin de ces micro-foyers, leur potentiel de croissance et de développement de métastases dans ce milieu d'ORN est incertain. La présence de nombreuses cellules inflammatoires, la dévascularisation, l'os nécrotique entourant les foyers cancéreux semblent être des conditions défavorables à la croissance tumorale. Notre hypothèse est une croissance tumorale ralentie par le milieu défavorable, pauvrement vascularisé. L'absence de reliquat et/ou de récidive chez ces patients semble l'étayer.

Toutes les séries, dont celle-ci, sont de petites cohortes, ne permettant pas de conclusions certaines. Cependant, nous avons mis en évidence une situation non décrite précédemment: la présence de foyers microscopiques de carcinome épidermoïde au sein d'ORN mandibulaire. Ces micro-foyers ont pu être mis en évidence grâce à une analyse histopathologique soigneuse. Nous avons soulevé le problème de la prise en charge de ces lésions tumorales découvertes fortuitement. La crainte du développement d'une nouvelle lésion maligne peut motiver une chirurgie extensive et mutilante mais ces données préliminaires semblent suggérer qu'une chirurgie trop agressive n'est pas nécessaire dans ce contexte. L'absence de récidive tumorale avec un suivi moyen de 35 mois corrobore cette hypothèse. 


\section{Conclusion}

La découverte de micro-foyers de carcinome épidermoïde au sein de mandibules atteintes d'ORN est rare et non décrite dans la littérature. Ceci motive une analyse anatomopathologique minutieuse de toute ostéoradionécrose mandibulaire réséquée. Jusqu'à présent, l'absence de données bibliographiques empêche de définir la prise en charge médicale et chirurgicale optimale chez des patients déjà fragilisés. Cette petite série encourage une approche thérapeutique minimaliste de cette pathologie, associée à un suivi renforcé. Les traitements chirurgicaux doivent être adaptés à l'évolutivité de l'ORN, et à l'état général du patient. 


\section{Références}

1. Marx RE. Osteoradionecrosis: a new concept of its pathophysiology. J. Oral Maxillofac. Surg. 1983;41:283-8.

2. Epstein J, van der Meij E, McKenzie M, et al. Postradiation osteonecrosis of the mandible: a long-term follow-up study. Oral Surg. Oral Med. Oral Pathol. Oral Radiol. Endod. 1997;83:657-62.

3. Marx RE, Johnson RP. Studies in the radiobiology of osteoradionecrosis and their clinical significance. Oral Surg. Oral Med. Oral Pathol. 1987;64:379-90.

4. Reuther T, Schuster T, Mende U, Kübler A. Osteoradionecrosis of the jaws as a side effect of radiotherapy of head and neck tumour patients--a report of a thirty year retrospective review. Int. J. Oral Maxillofac. Surg. 2003;32:289-95.

5. Thorn JJ, Hansen HS, Specht L, Bastholt L. Osteoradionecrosis of the jaws: clinical characteristics and relation to the field of irradiation. J. Oral Maxillofac. Surg. 2000;58:108893-5.

6. Harris M. The conservative management of osteoradionecrosis of the mandible with ultrasound therapy. Br. J. Oral Maxillofac. Surg. 1992;30:313-8.

7. Lyons AJ, West CM, Risk JM, et al. Osteoradionecrosis in head-and-neck cancer has a distinct genotype-dependent cause. Int. J. Radiat. Oncol. Biol. Phys. 2012;82:1479-84.

8. Meyer I. Infectious diseases of the jaws. J. Oral Surg. 1970;28:17-26.

9. Robard L, Louis M-Y, Blanchard D, Babin E, Delanian S. Medical treatment of osteoradionecrosis of the mandible by PENTOCLO: preliminary results. Eur. Ann. Otorhinolaryngol. Head Neck Dis. 2014;131:333-8.

10. Delanian S, Lefaix JL. [Mature bone radionecrosis: from recent physiopathological 
knowledge to an innovative therapeutic action]. Cancer Radiother. 2002;6:1-9.

11. Marx RE, Kline SN, Johnson RP, et al. The use of freeze-dried allogeneic bone in oral and maxillofacial surgery. J. Oral Surg. 1981;39:264-74.

12. Nabil S, Samman N. Risk factors for osteoradionecrosis after head and neck radiation: a systematic review. Oral Surg. Oral Med. Oral Pathol. Oral Radiol. 2012;113:54-69.

13. Beadle BM, Liao K-P, Chambers MS, et al. Evaluating the impact of patient, tumor, and treatment characteristics on the development of jaw complications in patients treated for oral cancers: a SEER-Medicare analysis. Head Neck. 2013;35:1599-605.

14. Peterson DE, Doerr W, Hovan A, et al. Osteoradionecrosis in cancer patients: the evidence base for treatment-dependent frequency, current management strategies, and future studies. Support. Care Cancer. 2010;18:1089-98.

15. Jacobson AS, Buchbinder D, Hu K, Urken ML. Paradigm shifts in the management of osteoradionecrosis of the mandible. Oral Oncol. 2010;46:795-801.

16. Zaghi S, Miller M, Blackwell K, et al. Analysis of surgical margins in cases of mandibular osteoradionecrosis that progress despite extensive mandible resection and free tissue transfer. Am. J. Otolaryngol. 2012;33:576-80.

17. Pitak-Arnnop P, Sader R, Dhanuthai K, et al. Management of osteoradionecrosis of the jaws: an analysis of evidence. Eur. J. Surg. Oncol. 2008;34:1123-34.

18. Cheriex KCAL, Nijhuis THJ, Mureau MAM. Osteoradionecrosis of the jaws: a review of conservative and surgical treatment options. J. Reconstr. Microsurg. 2013;29:69-75.

19. Bak M, Jacobson AS, Buchbinder D, Urken ML. Contemporary reconstruction of the mandible. Oral Oncol. 2010;46:71-6.

20. Urken ML, Buchbinder D, Costantino PD, et al. Oromandibular reconstruction using microvascular composite flaps: report of 210 cases. Arch. Otolaryngol. Head. Neck Surg. 
1998;124:46-55.

21. Chang EI, Leon P, Hoffman WY, Schmidt BL. Quality of life for patients requiring surgical resection and reconstruction for mandibular osteoradionecrosis: 10-year experience at the University of California San Francisco. Head Neck. 2012;34:207-12.

22. Jacobson AS, Zevallos J, Smith M, et al. Quality of life after management of advanced osteoradionecrosis of the mandible. Int. J. Oral Maxillofac. Surg. 2013;42:1121-8. 


\section{Figure}

Carcinome épidermoïde au sein de l'ostéonécrose (ORN) mandibulaire. Coloration hématoxyline-éosine, 10x.
A : micro-foyer de carcinome épidermoïde
B : ORN: paucicellulaire, fibrose
C: germes
D : cellules inflammatoires 
Table 1: Caractéristiques des patients

\begin{tabular}{|c|c|c|c|}
\hline Patient & Site & Stade & Traitement \\
\hline 1 & Oropharynx & III & $\begin{array}{c}\text { Chirurgie } \\
\text { RTCT adjuvante }\end{array}$ \\
\hline 2 & Cavité orale & IV & $\begin{array}{l}\text { CT néoadjuvante } \\
\text { Chirurgie } \\
\text { RTCT adjuvante }\end{array}$ \\
\hline 3 & Oropharynx & IV & $\begin{array}{c}\text { CT néoadjuvante } \\
\text { Chirurgie } \\
\text { RT adjuvante }\end{array}$ \\
\hline 4 & Cavité orale & IV & $\begin{array}{c}\text { CT néoadjuvante } \\
\text { Chirurgie } \\
\text { RT adjuvante }\end{array}$ \\
\hline 5 & Oropharynx & III & $\begin{array}{c}\text { CT néoadjuvante } \\
\text { Chirurgie } \\
\text { RT adjuvante }\end{array}$ \\
\hline
\end{tabular}


Table 2 : Traitements de l'ORN

délai et suivi sont en mois.

CE : carcinome épidermoïde

NI : non-interruptrice

I : interruptrice

LL : lambeau libre

\begin{tabular}{|c|c|c|c|c|c|}
\hline Patient & $\begin{array}{c}\text { Délai d'apparition de } \\
\text { l'ORN après RT }\end{array}$ & Biopsie & Type de Chirurgie & Anatomopathologie & Suivi \\
\hline 1 & 42 & CE & Mandibulectomie NI & ORN & 24 \\
\hline 2 & 12 & CE & Mandibulectomie NI & ORN & 30 \\
\hline 3 & 60 & CE & $\begin{array}{c}\text { Mandibulectomie I } \\
+ \text { LL }\end{array}$ & ORN & 33 \\
\hline 4 & 38 & ORN & $\begin{array}{c}\text { Mandibulectomie I } \\
+ \text { LL }\end{array}$ & CE & 42 \\
\hline 5 & 60 & ORN & Mandibulectomie I & CE & 46 \\
\hline
\end{tabular}




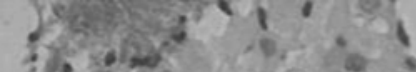

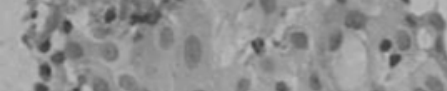

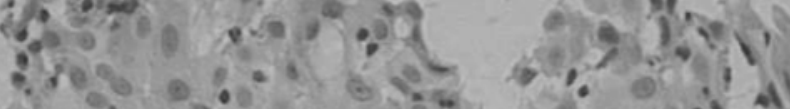

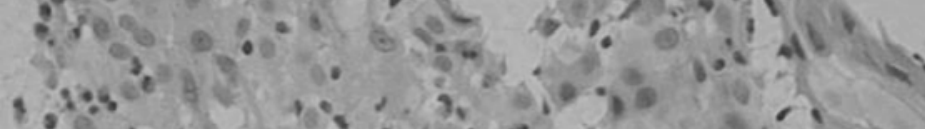

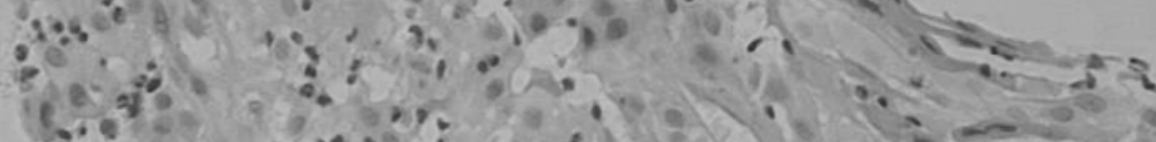

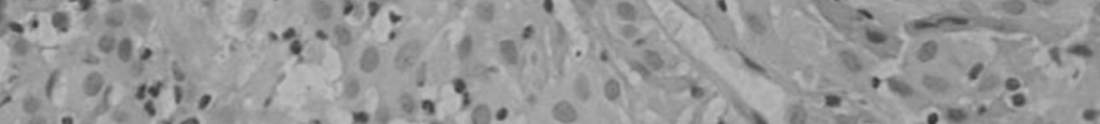

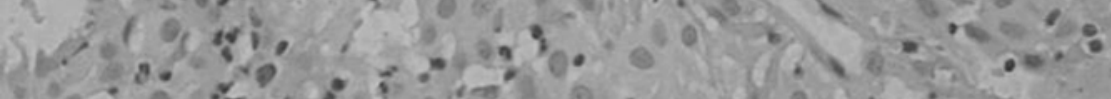

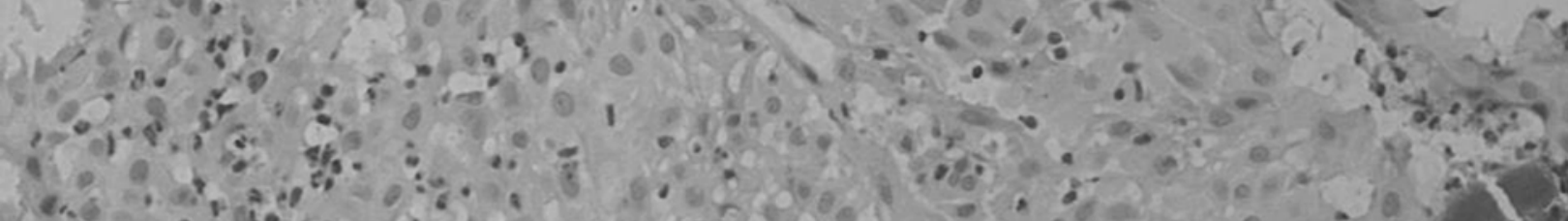

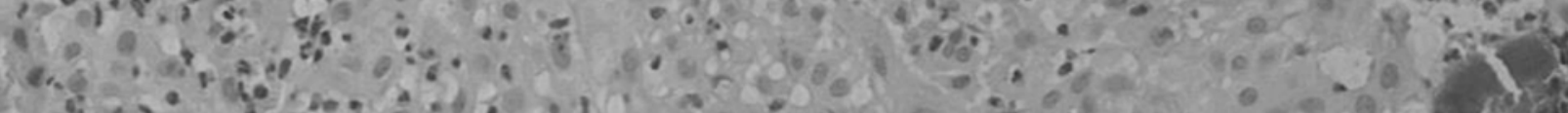

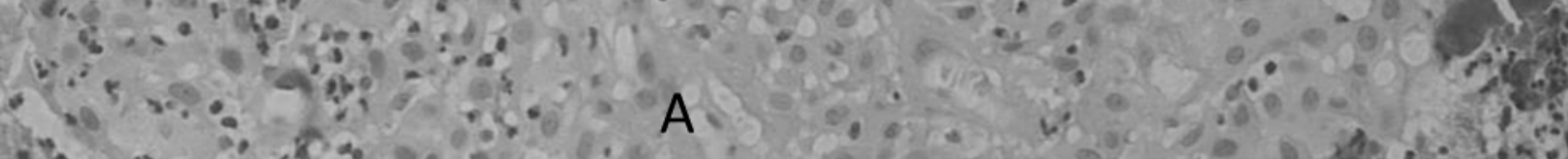

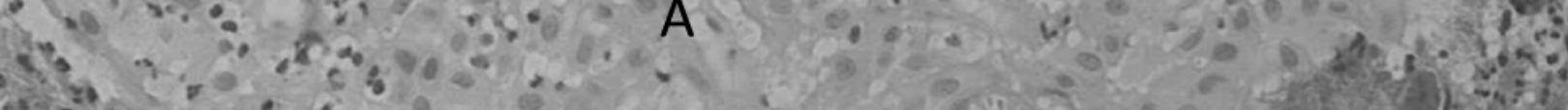
is

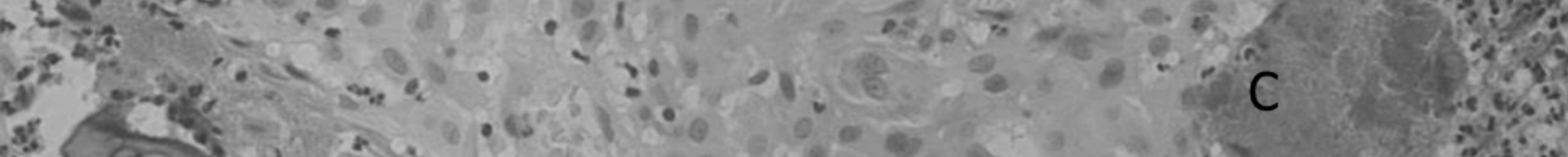

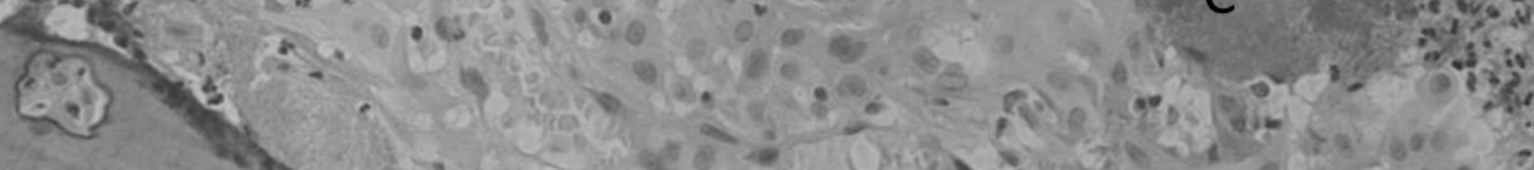

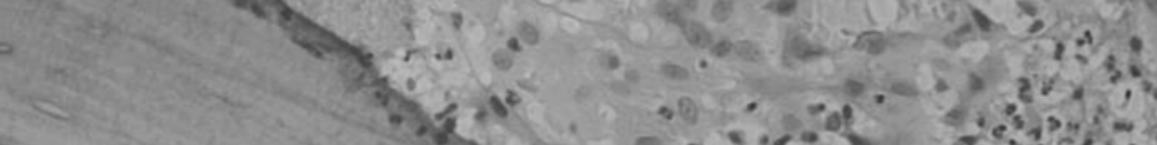

B $\quad 3.40^{2}$.

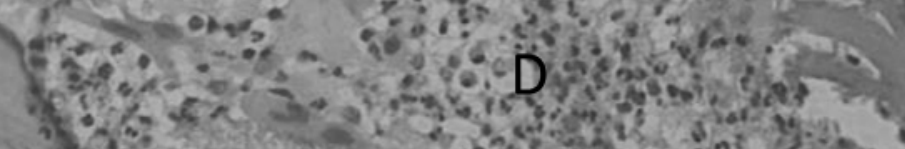

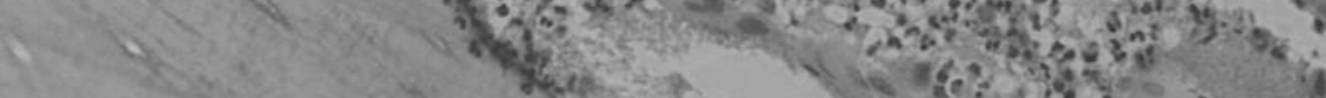

